\title{
A survey of primary care physician practices in antibiotic prescribing for the treatment of uncomplicated male gonoccocal urethritis
}

\author{
Alessandra Falchi ${ }^{1,2^{*}}$, Andrea Lasserre ${ }^{1,2}$, Anne Gallay $^{3}$, Thierry Blanchon ${ }^{1,2}$, Patrice Sednaoui ${ }^{4}$, François Lassau ${ }^{5}$, \\ Veronique Massari ${ }^{1,2}$, Clément Turbelin ${ }^{1,2}$ and Thomas Hanslik ${ }^{1,6,7}$
}

\begin{abstract}
Background: The development of resistance to antimicrobial therapy by Neisseria gonorrhoeae causes on-going problems for individual case management of gonorrhoea. Surveillance data about N. gonorrhoeae have indicated an increase in the incidence of gonorrhoea in France in 2006. As a consequence of the development of antibiotic resistance in N. gonorrhoeae, French guidelines excluded fluoroquinolones as a standard treatment for $\mathrm{N}$. gonorrhoeae. Ceftriaxone became the recommended treatment, associated with azithromycin for Clamydia trachomatis infection. Our aim was to describe the practice patterns of general practitioners (GPs) in managing the antibiotic treatment of patients with symptoms suggestive of uncomplicated male urethritis.
\end{abstract}

Methods: We developed a clinical vignette describing a man with typical gonococcal urethritis symptoms to elicit questions about antibiotic treatment. We mailed the electronic questionnaire to a random sample of 1000 French GPs belonging to the Sentinelles Network.

Results: By the end of the survey period, 350 vignettes were received, yielding a response rate of 35\%. Sixty-six GPs (20.2\%) prescribed the recommended antibiotics for the simultaneous treatment of N. gonorrhoeae and C. trachomatis infections, while 132 GPs (40.4\%) prescribed only non-recommended antibiotics, including ciprofloxacin in 69 cases $(21.1 \%)$. General practitioners with less than 10 years in practice showed better compliance to guidelines than those with more years in practice $(p<0.05)$.

Conclusions: The results suggest a mismatch between the guidelines and the antibiotic treatment of male uncomplicated urethritis by French GPs, mostly among the subgroup of physicians who have been in practice longer. Educational approaches based on practice feedback need to be developed to improve these deficits in the quality of care.

\section{Background}

Over the last few years, the number of gonorrhoea cases, caused by the gram negative bacterium Neisseria gonorrhoeae, has continually increased in many European countries, the United States and China [1-3]. However, over the past 60 years $N$. gonorrhoeae has developed resistance to multiple classes of antimicrobials. Since the first report of tetracycline resistance in 1985, gonococci that are resistant to tetracycline have spread globally [4]. During a sentinel surveillance study

\footnotetext{
* Correspondence: falchi@u707.jussieu.fr

'INSERM, UMPC UMR-S 707, F-75012, Paris, France

Full list of author information is available at the end of the article
}

in Western Europe in 2004, a total of 1055 gonoccocal isolates were analysed of which $21.3 \%$ showed resistance to penicillin [5]. In addition, high levels of ciprofloxacinresistant strains were reported worldwide in the early 1990s [6].

In France, an increase in the incidence of gonorrhoea cases has been indicated since 1998 [7]. In 2006, the number of gonorrhoea cases increased by more than $50 \%$ compared to 2005. Resistance of N. gonorrhoeae to ciprofloxacin sharply increased from $0.70 \%$ in 1998 to $43 \%$ in $2006[7,8]$.

An essential element in gonorrhoea control is the availability and provision of appropriate, effective

\section{Biomed Central}


antimicrobial therapy. Effective treatment not only eradicates infection in the affected individual and prevents the development of complications, but it also has an important public health benefit of decreasing transmission and eliminating reservoirs of infection. Thus, improving the appropriateness of antimicrobial use for the treatment of gonorrhoea is an important goal at national and international levels. New guidelines rationalizing antibiotic prescription for the probabilistic treatment of uncomplicated male urethritis were published in France in 2005 by the French Health Products Safety Agency (Afssaps) [9], recommending ceftriaxone as the first-line treatment, always associated with azithromycin for Clamydia trachomatis infection. Briefly, the recommended regimens, for all adult and adolescent patients, are ceftriaxone (intravenous/intramuscular) or cefixime (oral dosage) only if injectable is refused or not possible to do. If the patient is allergic to ceftriaxone or cefixime an alternative regimen is spectinomycin. Recommended regimens for C. trachomatis are azytromicin or doxycycline. As a consequence of the development of antibiotic resistance in $N$. gonorroheae, new French guidelines excluded fluoroquinolones as a standard treatment for $N$. gonorrhoeae.

General Practitioners (GPs) play an important role in preventing and treating male gonoccocal urethritis [10]. Consequently, it is worth making sure that GPs have a good working knowledge of antimicrobial use for gonorrhoea treatment. In this study, we conducted a national cross-sectional study using a clinical vignette to examine GPs' antibiotic prescribing in the treatment of gonoccocal male urethritis.

\section{Methods}

From June 2008 to August 2008, a cross-sectional study was conducted among GPs belonging to the Sentinelles Network [11], a computerized disease-surveillance system with about 1260 active volunteer GPs located throughout mainland France. The GPs participating in the Sentinelles Network are representative of the whole French GP population regarding age, location, type (rural/urban) and kind of practice (alone/pluridisciplinary) [12].

General practitioners who had previously agreed to participate at least once in our ad-hoc epidemiological studies by electronic means $(n=1000)$ were invited to complete a questionnaire

In September 2008, after data collection, a sample of 70 randomly selected non-respondent GPs was contacted by e-mail to ascertain the reasons for nonparticipation.

The French Sentinelles Network were given ethical approval (CNIL 471393) for the study. Moreover, the participation of GPs was voluntary and they could withdraw at any time. All data were handled confidentially and the results from the study were presented in a nonidentifiable way.

\section{Survey development}

A scientific committee composed of one specialist in venereal diseases, one epidemiologist, one general internist and one microbiologist developed a vignette $[13,14]$ designed to examine current practice patterns in the antibiotic treatment of uncomplicated male urethritis using scientific literature and guidelines $[9,13,14]$. The vignette was developed using a standardized protocol $[13,14]$. This approach has been shown to be an effective and cost-efficient method for measuring physicians' clinical decision making $[13,14]$. A pilot study was conducted with eight volunteer GPs of the Sentinelles Network that validated the vignette.

The scientific committee defined a master criteria list to review GP responses using scientific literature and guidelines [9,13-15]. We submitted the candidate criteria to a panel of 12 academic and community physicians of various specialities (general practice, infectious diseases, venereal diseases, microbiologists and public health). Based on their recommendations and group consensus, we finalized a master criteria list. Responses were considered appropriate if the hypothetical patient was treated simultaneously for N. gonorrhoeae and C. trachomatis infections with the following recommended firstline antibiotics:

a) ceftriaxone (250 to $500 \mathrm{mg}$ intravenously or intramuscularly, in a single dose) or cefixime (400 mg orally in a single dose) or, as an alternative regimen, spectinomycin (2 g intramuscularly in a single dose) for $N$. gonorrhoeae treatment

b) azithromycin (1 g orally in a single dose) or doxycycline (100 mg orally twice a day for 7 days) for $C$. trachomatis treatment.

The vignette was submitted to the GPs via a webbased electronic format (see Additional file 1): the GPs were asked to write a description of their treatment plan in an open-ended format. A time constraint of $20 \mathrm{~min}$ utes was imposed for completing the vignette. After answering a given section, participants could not return to the previous section to revise their answers.

After the clinical vignette, a new section measuring the GPs' knowledge of French guidelines and of the increasing resistance to ciprofloxacin for gonorrhoea treatment through two questions completed the survey:

1. "Are you aware of the increasing rate of ciprofloxacin resistance for gonorrhoea?"

2. "Are you aware of the existence of the new guidelines for the management of urethritis?" 
At the end of the study, each participating GP received an individual report of his results, the average performance of the group and the responses to the vignette.

\section{Statistical analysis}

Data were analysed using the $\mathrm{R}$ software program (http://www.r-project.org) using descriptive statistics for frequencies and chi-square tests for the comparisons of independent proportions, with Fisher's tests where appropriate. The level of significance was set as $\mathrm{p}<0.05$.

The GPs' answers were reviewed by two independent reviewers. An inter-rater reliability analysis using the Kappa statistic was performed to determine consistency among the raters [16]. The inter-rater reliability for the raters was found to be Kappa $=0.83(\mathrm{p}<0.001)$, suggesting a very good agreement.

\section{Results}

\section{Sample}

By the end of the survey period, 350 vignettes were received, yielding a response rate of $35 \%$. The vignette was fully completed by 327 GPs (93.4\%).

The responders were predominantly male $(83.4 \%)$ with a mean age of $52 \pm 7.5$ years $(\min =29 ; \max =69)$. Their practices were mainly located in urban zones (60\%) (Table 1).

Demographic characteristics (age, sex and location) of the GP responders, non-responders and of the GPs of the Sentinelles Network are shown in Table 1.

Among the 70 non-responding GPs, 67\% $(\mathrm{n}=47)$ declared that they had no experience of urethritis management in their practice, $26 \%(\mathrm{n}=18)$ did not participate because of a lack of time and $7 \%(n=5)$ did not participate for other several reasons (e.g. holidays, the survey was too complex or they were not interested.

\section{Antimicrobial agents used}

Sixty-six GPs (20.2\%) prescribed the simultaneous administration of the recommended antibiotics for $N$. gonorrhoeae and C. trachomatis infections (Table 2) for the hypothetical patient.
Table 2 Antibiotic treatments prescribed by the GPs

\begin{tabular}{|c|c|}
\hline Treatments prescribed & Respondents No. (\%) \\
\hline \multicolumn{2}{|l|}{ No $=327(\%)$} \\
\hline Two recommended antibiotics* & $66(20.2)$ \\
\hline One recommended antibiotic & $114(34.8)$ \\
\hline against N. gonorrhoeae $\boldsymbol{\Psi}^{\boldsymbol{\Psi}}$ & $69(21.1)$ \\
\hline against C. trachomatis $\boldsymbol{\Psi} \boldsymbol{\Psi}$ & $45(13.7)$ \\
\hline One non-recommended antibiotic & $147(45)$ \\
\hline Ciprofloxacin & $69(21.1)$ \\
\hline Amoxicillin & $24(7.3)$ \\
\hline Others & $54(16.6)$ \\
\hline
\end{tabular}

${ }^{*}$ for N. gonorrhoeae and C. trachomatis co-infections

${ }^{\Psi}$ Ceftriaxone or spectinomycin or cefixime

${ }^{4 \psi}$ Azithromycin or doxycycline

One hundred and fourteen (34.8\%) GPs prescribed only one recommended antibiotic against $N$. gonorrhoeae $(\mathrm{n}=69)$ or against $C$. trachomatis $(\mathrm{n}=45)$. One hundred and thirty-two GPs (40.4\%) prescribed only non-recommended antibiotics, including ciprofloxacin, in 69 cases (Table 2).

Knowledge of fluoroquinolone resistance emergence and the treatment guidelines

When asked about the fluoroquinolone resistance of $N$. gonorrheoae, $26 \%(n=84)$ of the respondents declared that they were aware of the high rate of resistance in France. The existence of the French guidelines was declared known by $25 \%(\mathrm{n}=81)$ of GPs. Among the 69 GPs who prescribed ciprofloxacin as first-line treatment, $84 \%(\mathrm{n}=58)$ were not aware of the emergence of fluoroquinolone resistance.

\section{Influence of prescriber factors}

General practitioners who had been in practice for 10 years or less were more likely to prescribe the recommended antibiotics than GPs with more years in practice ( $p<0.0001$; Table 3). General practitioners who had been in practice for 10 years or less were also more likely to be aware of the high rate of fluoroquinolone resistance and of the existence of guidelines ( $\mathrm{p}<0.0001$; Table 3 ). Gender and geographic location (urban, suburban or

Table 1 Demographic characteristics of GP respondents, non-respondents and GPs of the Sentinelles Network

\begin{tabular}{lcccc}
\hline Demographic characteristics & $\begin{array}{c}\text { GP respondents } \\
\text { No }=\mathbf{3 5 0}\end{array}$ & $\begin{array}{c}\text { GP non respondents } \\
\text { No }=\mathbf{6 5 0}\end{array}$ & $\begin{array}{c}\text { GPs of the Sentinelles } \\
\text { Network No }=\mathbf{1 2 6 0}\end{array}$ & $\begin{array}{c}\mathbf{p} \text { value } \\
\text { Sex (male) } \%\end{array}$ \\
\hline Age (years), mean \pm SD & $52 \pm 7.5$ & $82 \%$ & $52.8 \pm 7.8$ & 0.97 \\
\hline Practice location, $\%$ & & $52.3 \pm 7.6$ & & \\
\hline Urban & $60 \%$ & $61 \%$ & $62 \%$ & 0.96 \\
\hline Sub-Urban & $19 \%$ & $16 \%$ & $18 \%$ & 0.96 \\
\hline Rural & $21 \%$ & $19 \%$ & $20 \%$ & 0.96 \\
\hline
\end{tabular}


Table 3 Association between years in practice and the physicians' practice patterns in managing gonoccocal urethritis treatment and their knowledge of fluoroquinolone resistance emergence and of the guidelines

\begin{tabular}{|c|c|c|c|c|}
\hline Years of practice & $\begin{array}{c}\leq 10 \text { years } \\
\text { No. }(\%)\end{array}$ & $\begin{array}{l}11-20 \text { years } \\
\text { No. (\%) }\end{array}$ & $\begin{array}{l}\text { >20years } \\
\text { No. (\%) }\end{array}$ & $\mathrm{p}$ value \\
\hline \multicolumn{5}{|l|}{ First-line treatment } \\
\hline One recommended antibiotic against $N$. gonorrhoea $e^{*}$ & $18(36)$ & $22(28)$ & $29(15)$ & 0.0012 \\
\hline One recommended antibiotic against C. trachomatis ${ }^{{ }^{*} \Psi}$ & $12(42)$ & $19(24)$ & $14(2.5)$ & 0.0002 \\
\hline Two antibiotics prescribed concomitantly* & $20(40)$ & $26(32)$ & $20(10)$ & $<0.0001$ \\
\hline \multicolumn{5}{|l|}{ Knowledge of fluoroquinolone resistance emergence and guidelines } \\
\hline Awareness of the existence of the French guidelines & $22(44)$ & $26(32.5)$ & $33(16.8)$ & $<0.0001$ \\
\hline Awareness of fluoroquinolone resistance & $28(56)$ & $24(30)$ & $32(16)$ & $<0.0001$ \\
\hline
\end{tabular}

rural) of the GP was not associated with the treatments prescribed or knowledge of the guidelines ( $\mathrm{p}>0.05)$.

\section{Discussion}

\section{Summary of the main findings}

In this study, only a minority of the GPs (20.2\%) correctly co-prescribed the first-line treatment recommended for $N$. gonorrhoeae and C. trachomatis co-infection. Moreover, the GPs frequently prescribed non-recommended first-line treatment, including ciprofloxacin, despite the high level of fluoroquinolone resistance of $N$. gonorrhoeae in France (43\% during 2006) [7,8].

The relatively low level of prescribed treatment against C. trachomatis could be explained by the description of the purulent discharge of the urethritis in the vignette, which is a typical sign of $N$. gonorrhoeae infection. These results are in agreement with a previous study on the compliance of emergency department practitioners with the Centers for Disease Control (CDC) recommendations for diagnosing and treating sexually transmitted diseases. This study showed that the recommended antibiotic regimens for $N$. gonorrhoeae and C. trachomatis were correctly co-prescribed in only one third of cases [17].

In this study, GPs who had been in practice for 10 years or less were more likely to prescribe first-line treatment in accordance with the guidelines and they were better informed about the increasing rate of fluoroquinolone resistance and about the new national strategy for the therapeutic management of probabilistic treatment for uncomplicated male urethritis than physicians who had been in practice longer.

The results suggested that the overall rate of compliance with national guidelines concerning antibiotic choice must be improved among GPs. In particular, the subgroups of physicians who have been in practice for longer are at risk of providing lower-quality care.

These findings are consistent with other studies that suggested an inverse relationship between years in practice and the quality of care provided $[18,19]$. A systematic review which evaluated the relationship between clinical experience and performance suggested that physicians who have been in practice for a longer period and those who are older possess less factual knowledge, are less likely to adhere to appropriate standards of care, and may also have poorer patient outcomes. These effects seemed to persist in studies that adjusted for other known predictors of quality, such as patient comorbidity and physician volume or specialization [18].

As observed in this study, barriers to guideline adherence, such as a lack of agreement and a lack of familiarity, seem improbable in the case of urethritis, for which the recent guidelines are easily justified (a high rate of quinolone resistance) and quite straightforward to apply (a simple antibiotic prescription) [7-9]. One reason for the clinicians prescribing contrary to the guidelines is most probably unawareness of the guidelines. The fact that an increasing number of years in practice is associated with prescribing non-recommended antibiotics favours this hypothesis, although we cannot exclude other reasons. For example, GPs may find that prescribing an intramuscular treatment such as ceftriaxone might create a barrier because the patient has to buy the injectable drug and then go back to the GP to be injected with it. Another possible reason for little knowledge of the guidelines could be due to the volume of biomedical information available and the time needed to learn about new developments. The guidelines rationalizing antibiotic prescription for the probabilistic treatment of uncomplicated male urethritis were published in France in 2005 and disseminated to GPs at the national level by Afssaps [9], preferably by electronic means (mailing lists and publication on health websites). The fact that the recommendations were preferentially disseminated using electronic sources, of which younger GPs are greater users than older practitioners, could have added another barrier against compliance with the 
recommendations. It must be noted that, according to a survey by the Centers for Disease Control and Prevention's National Center for Health Statistics [20], it seems that a majority of office-based physicians use electronic medical record (EMRs) systems. A 2010 report by the Centers for Disease Control and Prevention's National Center for Health Statistics [20] reported that the younger the doctor, the more likely he or she was to embrace electronic medical records (EMRs), although the resistance to them by older physicians seems to have decreased as EMRs have become more common in offices and hospitals.

\section{Limitations of the study}

There were several limitations to this study. The first was that we used a self-reported survey to measure compliance with guideline recommendations as it is possible that physicians answered questions in ways that reflected professional desirability rather than their own practices. The second limitation of this study was that we only proposed one scenario that did not cover the full clinical presentation of urethritis. However, the principal objective was to evaluate the application of guideline recommendations for the management and treatment of male uncomplicated urethritis, mostly due to gonorrhoea. Thirdly, there may have been limitations in generalizability to the French GP population, as reflected by the response rate of $35 \%$. Moreover, the GPs who participated in this study were more concerned about urethritis than non-responders, as shown by the non-responders' answers, and therefore their practices might differ. However, we found no differences in the demographic data between responders and non-responders regarding age, gender, and location of the practice.

\section{Conclusions}

\section{Implications for clinical practice and future research}

This study suggests that interventions to improve physicians' knowledge about the management of public health issues are warranted, especially regarding the subgroup of physicians who have been in practice the longest. Moreover, because drug-resistant $N$. gonorrhoeae is becoming an increasing problem worldwide, such an evaluation of treatment practices would probably be useful in other countries.

\section{Additional material}

\section{Additional file 1:}

\section{Acknowledgements}

The authors thank all of the general practitioners of the Sentinelles Network who participated and all of the experts who helped develop the vignette.

\section{Funding}

The Institut de Veille Sanitaire (InVS, France) provided funding and support for the design and conduct of the study, interpretation of the data and approval of the manuscript.

\section{Author details}

${ }^{1}$ INSERM, UMPC UMR-S 707, F-75012, Paris, France. ${ }^{2}$ UPMC Université Paris 06, UMR-S U707, F-75012, Paris, France. ${ }^{3}$ Institut de Veille Sanitaire, St Maurice, Paris, France. ${ }^{4}$ Institut Alfred Fournier Paris, France. ${ }^{5}$ Hôpital SaintLouis, AP-HP, Paris, France. ${ }^{6}$ Université Versailles Saint Quentin en Yvelines, F78000, Versailles, France. ${ }^{7}$ Assistance Publique Hôpitaux de Paris, Service de Médecine Interne, Hôpital Ambroise Paré, F-92100, Boulogne Billancourt, France.

\section{Authors' contributions}

All authors read and approved the final manuscript. AF collected and analysed the data, conceived this paper and took primary responsibility for writing it. AL contributed to the study design, collected and analysed the data and reviewed the paper. AG was the principal investigator on the project and took part in the writing and review of the paper. TB contributed to the study design and reviewed the paper. PS contributed to the study design and reviewed the paper. FL contributed to the study design and reviewed the paper. VM contributed to the study design and reviewed the paper. $C T$ contributed to the study design, designed the informatics support and reviewed the paper. $\mathrm{TH}$ conceptualized and wrote the proposal for the vignette, contributed to the study design and took part in the writing and review of the paper.

\section{Competing interests}

The authors declare that they have no competing interests.

Received: 1 October 2010 Accepted: 18 May 2011

Published: 18 May 2011

\section{References}

1. Fenton KA, Lowndes CM: Recent trends in the epidemiology of sexually transmitted infections in the European Union. Sex Transm Infect 2004, 80:255-263.

2. Liao MK, Bell WM, Gu Y, Yang NF, Eng W, Fu L, Wu CG, Zhang Y, Chen AM, Jolly Dillon JA: Clusters of circulating Neisseria gonorrhoeae strains and association with antimicrobial resistance in Shanghai. J Antimicrob Chemother 2008, 61:478-487.

3. Kolader ME, Dukers NHTM, van der Bij AK, Dierdorp M, Fennema JSA, Coutinho RA, Bruisten SM: Molecular epidemiology of Neisseria gonorrhoeae in Amsterdam, the Netherlands, shows distinct heterosexual and homosexual networks. J Clin Microbiol 2006, 44:2689-2697.

4. Starnino S, Neri A, Stefanelli P: Molecular analysis of tetracycline-resistant gonococci: rapid detection of resistant genotypes using a real-time PCR assay. FEMS Microbiol Lett 2008, 286:16-23.

5. Martin IM, Hoffmann S, Ison CA: European Surveillance of Sexually Transmitted Infections (ESSTI): the first combined antimicrobial susceptibility data for Neisseria gonorrhoeae in Western Europe. J Antimicrob Chemother 2006, 58:587-593.

6. Tapsall JW, Limnios EA, Murphy DM, on behalf of the Australian Gonococcal Surveillance Programme: An analysis of trends in antimicrobial resistance in Neisseria gonorrhoeae isolated in Australia, 1997-2006. J Antimicrob Chemother 2008, 61:150-155.

7. Gallay A, Bouyssou-Michel A, Lassau F, Basselier B, Sednaoui P, Laboratoires du Réseau Renago: Neisseria gonorrhoeae infections in France in 2006: significant progression in women and persistent increase of ciprofloxacin resistance. Bull Epidémiol Hebdomadaire 2008, 5-6:33-36, (in French).

8. Monfort L, Caro V, Devaux Z, Delannoy AS, Brisse S, Sednaoui P: First neisseria gonorrhoeae genotyping analysis in france: identification of a strain cluster with reduced susceptibility to Ceftriaxone. J Clin Microbiol 2009, 47:3540-3545.

9. Agence française de sécurité sanitaire des produits de santé (Afssaps): Antibiotherapy applied to uncomplicated urethritis and cervicitis. Médecine et maladies infectieuses 2006, 36:27-35. 
10. Massari $V$, Dorléans $Y$, Flahault A: Persistent increase in the incidence of acute male urethritis diagnosed in general practices in France. $\mathrm{Br} J \mathrm{Gen}$ Pract 2006, 56:110-114.

11. Flahault A, Blanchon T, Dorléans Y, Toubiana L, Vibert JF, Valleron AJ: Virtual surveillance of communicable diseases: a 20-year experience in France. Stat Methods Med Res 2006, 15:413-421.

12. Chauvin P, Valleron AJ: Attitude of French general practitioners to the public health surveillance of communicable diseases. Int J Epidemiol 1995, 24:435-440.

13. Peabody JW, Luck J, Glassman P, Dresslhaus TR, Lee M: Comparison of vignettes, standardized patients and chart abstraction. A prospective validation study of 3 methods for measuring quality. JAMA 2000, 283:1715-1721.

14. Peabody JW, Luck J, Glassman P, Jain S, Hansan J, Spell M: Measuring the quality of physician practice by using clinical vignettes: A prospective validation study. Ann Intern Med 2004, 141:771-781.

15. CDC: Update to CDC's Sexually Transmitted Diseases Treatment Guidelines, 2006: Fluoroquinolones no longer recommended for treatment of gonococcal infections. MMWR 2007, 56:332-336.

16. Landis JR, Koch GG: The measurement of observer agreement for categorical data. Biometrics 1977, 33:159-174.

17. Kane BG, Degutis LC, Sayward HK, D'Onofrio G: Compliance with the Centers for Disease Control and Prevention recommendations for the diagnosis and treatment of sexually transmitted diseases. Acad Emerg Med 2004, 11:371-377.

18. Choudhry NK, Fletcher RH, Soumerai SB: Systematic review: the relationship between clinical experience and quality of care. Ann Intern Med 2005, 142:260-273.

19. Doroodchi H, Abdolrasulnia M, Foster JA, Foster E, Turakhia MP, Skelding KA, Sagar K, Casebeer LL: Knowledge and attitudes of primary care physicians in the management of patients at risk for cardiovascular events. BMC Fam Pract 2008, 9:42.

20. Hsiao CJ, Beatty PC, Hing E, Woodwell DA, Rechtsteiner EA, Sisk JE: Electronic medical record/electronic health record use by office-based physicians: United States, 2008 and preliminary 2009. National Center for Health Statistics Health E-stat 2009.

\section{Pre-publication history}

The pre-publication history for this paper can be accessed here: http://www.biomedcentral.com/1471-2296/12/35/prepub

doi:10.1186/1471-2296-12-35

Cite this article as: Falchi et al.: A survey of primary care physician practices in antibiotic prescribing for the treatment of uncomplicated male gonoccocal urethritis. BMC Family Practice 2011 12:35.

\section{Submit your next manuscript to BioMed Central and take full advantage of:}

- Convenient online submission

- Thorough peer review

- No space constraints or color figure charges

- Immediate publication on acceptance

- Inclusion in PubMed, CAS, Scopus and Google Scholar

- Research which is freely available for redistribution

Submit your manuscript at www.biomedcentral.com/submit 